\title{
CRYSTALLO-CO-AGGLOMERATION: A NOVEL TECHNIQUE TO IMPROVE FLOW AND COMPRESSIBILITY
}

\author{
Rahate Nikita B.*, Bodhankar Mitali M., Dhoke Priyanka N. \\ Department of Pharmaceutics, Gurunanak College of Pharmacy, Near Dixit Nagar, Behind C.P.Foundary, Nagpur, India \\ *Corresponding Author's E-mail: mit21_bod@yahoo.co.in
}

\begin{abstract}
:
Crystallo-co-agglomeration is a particle design technique in which a drug is crystallized and agglomerated with an excipient or another drug, which may or may not be crystallized in same system. The various parameters optimized were type, amount and mode of addition of bridging liquid, temperature and agitation speed. They were characterized for micrometritic properties (particle size and shape, flowability, packability, bulk density, wettability and compressibility). The prepared spherical crystals with different polymers exhibited excellent physicochemical properties like flowability, pack ability and wettability compared with the pure drug. The process is simple and inexpensive enough for scaling upto a commercial level. It reduces time and cost by enabling faster operation, less machinery and fewer personnel.
\end{abstract}

Keywords: Crystallo-co-agglomeration, flowability, compressibility, bioavailability.

\section{INTRODUCTION:}

Today the tablet is the most popular dosage form of all pharmaceutical preparations produced. From the manufacturing point of view tablets can be produced at much higher rate than any other dosage form ${ }^{1}$. The material used for the production of tablet should be in physical form that flows smoothly, directly compressible and physically stable so as to achieve rapid production capability of tablet formulation. Direct compression is the most efficient process used in tablet manufacturing because it is the fastest, simplest and least expensive tablet-compression procedure. Many processing steps mainly granulation and drying are omitted in direct compression, and additionally, wet technology cannot be used with sensitive agent (e.g., in effervescent tablet making). Direct tableting of pharmaceutical materials is desirable to reduce the cost of production. However, compressing a drug directly requires good micromeritic properties, such as flowability, and a good and reproducible compression behavior. To impart these properties the drugs are subjected to particle design techniques, spherical crystallization is one the techniques of particle design ${ }^{2-4}$.

In the pharmaceutical field, Kawashima et al. have given impulse to the research of the spherical crystallization process. "Spherical crystallization" was defined by Kawashima as "an agglomeration technique that transforms crystals directly into a compacted spherical form during the crystallization process." Spherical crystallization is a particle design technique which is restricted to only water insoluble single large dose drugs only because several excipients are hydrophilic in nature hence addition of these excipients in the agglomerates with help of organic bridging liquid is difficult. ${ }^{5}$

Kadam et al developed the crystallo-co-agglomeration (CCA) technique, which is a modification of spherical crystallization. CCA has been designed to overcome the limitations of spherical crystallization to obtain directly compressible agglomerates. ${ }^{6,7}$ Basically, it's single step process used for size enlargement of single, two or more, small dose or large dose drugs, in combination with or without diluents. The process of CCA involves simultaneous crystallization and agglomeration of drug/s with/without excipients from good solvent and/or bridging liquid by addition of a non-solvent. ${ }^{8,9}$

The tablet dosage form prepared from agglomerated crystals showed improvements in tensile strength, hardness, friability, disintegration time and dissolution rate as compared to tablets prepared from granules. Thus this technique is simple, less expensive, may be an advantages for developing it on a commercial scale for manufacturing of tablets. ${ }^{10}$

\section{METHODS OF SPHERICAL CRYSTALLISATION:}

The methods of spherical crystallization are categorized

\section{Spherical Agglomeration ${ }^{11}$}

A near saturated solution of the drug in the good solvent is poured into the poor solvent. Provided that the poor and good solvents are freely miscible and the affinity between the solvents is stronger than the affinity between the drug and the good solvent, crystals will precipitate immediately. In the spherical agglomeration method also a third solvent called the bridging liquid is added in a smaller amount to promote the formation of agglomerates. Under agitation, the bridging liquid (the wetting agent) is added. The bridging liquid should not be miscible with the poor solvent and should preferentially wet the precipitated crystals. As a result of interfacial tension effects and capillary forces, the bridging liquid act to adhere the crystals to one another. The SA method has been applied to several drugs, and it has been found that the product properties are quite sensitive to the amount of the bridging liquid. Less than the optimum amount of bridging liquid produces plenty of fines and more than optimum produces very coarse particles. Also the choice of bridging liquid, the stirring speed and the concentration of solids (or of the 
solute) are of importance. In the case of lactose, the agglomerate size distribution was affected by both the size of raw particles and the amount of bridging liquid used. At increasing stirring rate the agglomeration was reduced because of increasing disruptive forces. Higher stirring rate produce agglomerates that are less porous and more resistant to mechanical stress, and the porosity decreases when the concentration of solid increases. The viscosity of the continuous phase has an effect on the size distribution of the agglomerates. The choice of bridging liquid has an influence on the rate of agglomeration and on the strength of the agglomerates.

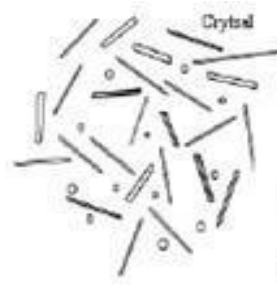

Formation of crystals

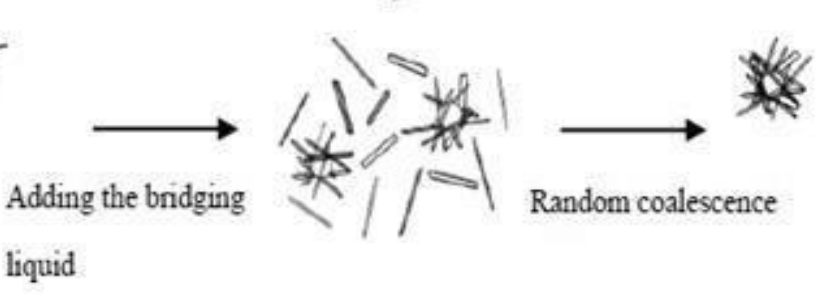

Precipitated crystals and

aggregate with bridging liquid
Spherical agglomerates

Enlarged spherical

agglomerates

Figure 1: Showing formation of agglomerates

\section{Quasi Emulsion Solvent Diffusion Method (QESD) ${ }^{11}$}

In the emulsion solvent diffusion the affinity between the drug and the good solvent is stronger than that of the good solvent and the poor solvent. The drug is dissolved in the good solvent, and the solution is dispersed into the poor solvent, producing emulsion (quasi) droplets, even though the pure solvents are miscible. The good solvent diffuses gradually out of the emulsion droplets into the surrounding poor solvent phase, and the poor solvent diffuses into the droplets by which the drug crystallizes inside the droplets. The method is considered to be simpler than the SA method, but it can be difficult to find a suitable additive to keep the system emulsified and to improve the diffusion of the poor solute into the dispersed phase.

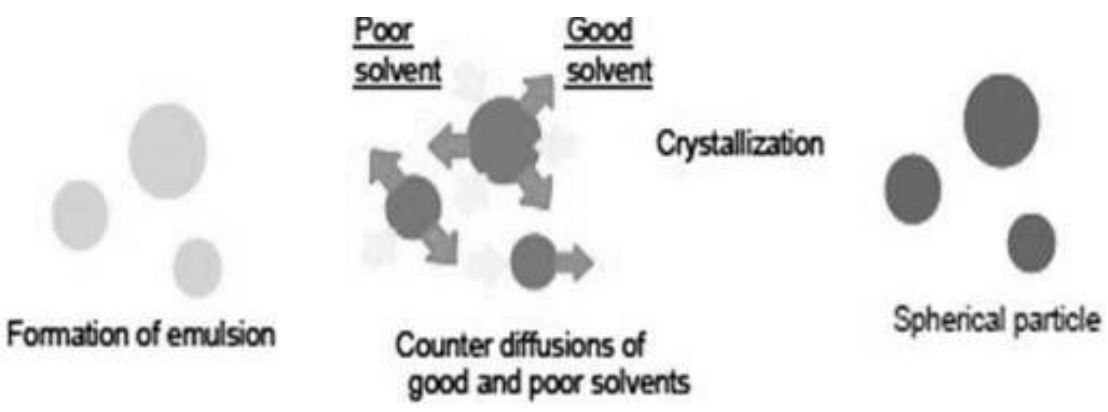

Figure 2: explaining Emulsion Solvent Diffusion Method

\section{Ammonia Diffusion Method (AD) ${ }^{12}$}

In this method, the mixture of three partially immiscible solvent i.e. acetone, ammonia water, dichloromethane was used as a crystallization system. In this system ammonia water acted as bridging liquid as well as good solvent,
Acetone was the water miscible but a poor solvent, thus Drug precipitated by solvent change without forming ammonium salt. Water immiscible solvent such as hydrocarbons or halogenated hydrocarbons e.g. dichloromethane induced liberation of ammonia water.

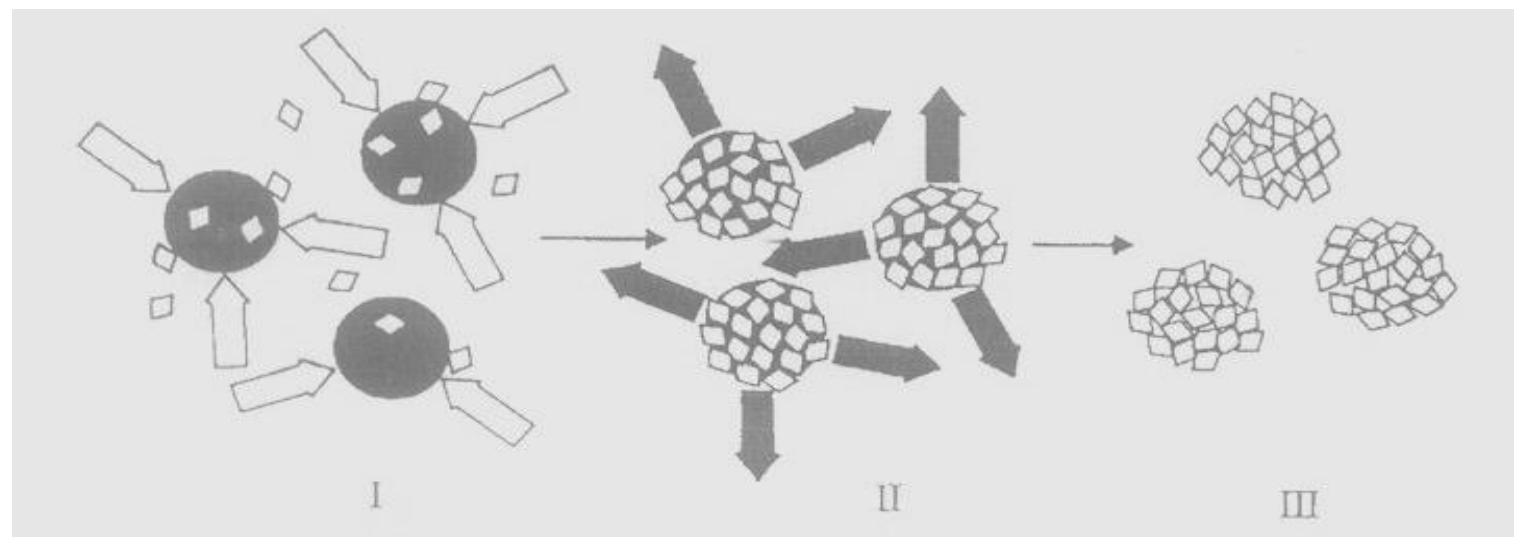

Figure 3: explaining Ammonia Diffusion Method (AD) 


\section{Neutralization Method $^{13}$}

In this method, the drug dissolved in the good solvent and placed in the cylindrical vessel with constant stirring. While stirring an aqueous polymer solution and one neutral solution was added which neutralize the good solvent and crystallize out the drug. Then bridging liquid was added drop wise at a definite rate, followed by agglomeration of the crystal form of the drug takes place.

\section{CRYSTAL-CO-AGGLOMERATION TECHNIQUE $(\mathrm{CCA})^{14}$}

It is a modification of the spherical crystallization technique in which drug is crystallized and agglomerated with an excipient or with another drug. This process enables design of agglomerates containing two drugs or poorly compressible drug in combination with diluents and is restricted to water insoluble large-dose drugs only. Difference in the physicochemical properties of drug molecules and excipient is a major challenge in the selection of the solvent system for the Crystal-coagglomeration technique.

\section{Steps involved in Crystal-co-agglomeration technique}

Drug+ good solvent

$\downarrow$ agitation

Bridging liquid (homogenous solution)

$\downarrow$ added continuous stirring

Bad solvent/polymeric solution

$\downarrow$ crystal formation

Spherical agglomeration

\section{ADVANTAGES OF CRYSTALLO-CO- AGGLOMERATION: ${ }^{15}$}

1. Spherical crystallization technique has been successfully utilized for improvement of flowability and compressibility of crystalline drug.

2. This technique could enable subsequent processes such as separation, filtration, drying etc. to be carried out more efficiently.

3. By using this technology, physicochemical properties of pharmaceutical crystals are dramatically improved for pharmaceutical process i.e. milling, mixing and tabletting because of their excellent flowability and packability.

4. This technique may enable crystalline form of a drug to be converted into different polymorphic form and thus attain better bioavailability.

5. For masking of the bitter taste of drug.

6. Preparation of microsponges, microspheres and nanaospheres, microbaloons, nanoparticles and micro pellets as novel particulate drug delivery system.

\section{FACTORS AFFECTING THE PROCESS OF AGGLOMERATION:}

\section{Diluents selection}

The use of diluent has been suggested in CCA for size enlargement of low dose drugs. Diluent selected must be physico-chemically and physiologically inert, and inexpensive. ${ }^{16}$ Moreover, it should be insoluble in the aqueous phase to avoid the losses through the (c) 2011, JDDT. All Rights Reserved continuous/external phase. Considering desired attributes, talc has been used as a diluent in the development of the CCA process. Talc as an excipient/diluent in bead/pellet making gets strengthened further. Recently, starch and Nastarch glycolate has been used in preparation of rapidly disintegrating agglomerates of naproxen by the CCA process. ${ }^{17,18}$

\section{Solvent system}

The solvent system selection for the CCA process depends on solubility and stability of drug/s. Since, majority of drugs are soluble in organic solvents and poorly soluble in water. Use of organic solvent (relatively nontoxic) has been recommended as a good solvent and or bridging liquid and water as an external/processing phase (nonsolvent). The bridging liquid should carry out preferential wetting of crystals/solids and form liquid bridges during the process of agglomeration, and simultaneously, it should be immiscible with a non-solvent. If bridging liquid is used as a good solvent, it means, it performs dual role of a good solvent and bridging liquid. The good solvent used should be volatile and immiscible with non-solvent to avoid drug loss due to co-solvency. Amount of bridging liquid required can be decided by the trial and error method or the ternary phase diagram. It has been observed that if addition of bridging liquid becomes inadequate, then it leads to generation of smaller size agglomerates with more percentage of fines. And, excess addition of bridging liquid generates bigger size agglomerates and requires more processing time for completion of the agglomeration process. ${ }^{19,20,21}$

\section{Mode and intensity of agitation:}

High speed agitation is necessary to disperse the bridging liquid throughout the system. Change in the agitation pattern or fluid flow will affect the shape of agglomerates. The extent of mechanical agitation and the concentration of bridging liquid determine the rate of formation of agglomerates and their final size. ${ }^{22}$

\section{Temperature of the system:}

It has a significant influence on the shape, size and texture of the agglomerates. The effect of temperature on spherical crystallization is probably due to its effect on the solubility of drug substance. ${ }^{22}$

\section{Residence time:}

It is defined as the time for which agglomerates remain suspended in the reaction mixture. Residence time affects the strength of agglomerates. ${ }^{23}$

\section{USE OF POLYMERS:}

Presence of additives like polymers and surface active agents whose surfaces are not similar to the crystal surfaces can influence molecular aggregation during crystallization. Studies have revealed that crystallization and agglomeration of pure drugs shows poor compressibility and handling qualities. Addition of polymers such as HPMC, PEG and PVP has improved the properties of spherical agglomerates. It has been reported that PVP improved the micromeritic properties, solubility and dissolution rate of spherical crystals. The agglomerates prepared using an optimum amount of HPMC has showed adequate sphericity and mechanical strength to the ISSN: 2250-1177 
agglomerates, whereas its excess addition imparts ellipticity and deformation to agglomerates. PEG causes reduction in the interfacial tension between water (external phase) and bridging liquid resulting into reduction in the force of cohesion between particles leading to generation of small size spherical agglomerates. ${ }^{24,25}$ PEG, due to its soft and plastic nature, undergoes plastic deformation and gives better compressibility to the agglomerates during the process of compression. Whereas, EC, due to its high yield strength, on crystallization in a non-solvent imparts more strength to the agglomerates. Its solubility in the good solvent and bridging liquid (organic solvent) imparts higher viscosity to the internal phase and results into increased interfacial tension. The increased viscosity retards the diffusion of bridging liquid, hampers nucleation, crystal formation, and increases the time required for completion of the agglomeration process. Hence, to get agglomerates having satisfactory sphericity and strength, and compacts having adequate tensile strength, a combination of HPMC, PEG, and EC has been recommended during the crystallo-co-agglomeration process. ${ }^{26,27}$

\section{THE PRINCIPLE STEPS INVOLVED IN THE PROCESS OF SPHERICAL CRYSTALLIZATION}

\section{Flocculation Zone: ${ }^{28-33}$}

In this zone, the bridging liquid displaces the liquid from the surface of the crystals and these crystals are brought in close proximity by agitation; the adsorbed bridging liquid links the particles by forming a lens bridge between them. In these zones, loose open flocs of particles are formed by pendular bridges.

\section{Zero Growth Zone:}

Loose floccules get transferred into tightly packed pellets, during which the entrapped fluid is squeezed out followed by squeezing of the bridging liquid onto the surface of small flocs causing poor space in the pellet of completely filled with the bridging liquid. The driving force for the transformation is provided by the agitation of the slurry causing liquid turbulence, pellet-pellet and pellet-stirrer collision.

\section{Fast Growth Zone:}

The fast growth zone of the agglomerates takes place when sufficient bridging liquid has squeezed out of the surface on the small agglomerates. This formation of large particles following random collision of well-formed nucleus is known as coalescence. Successful collision occurs only if the nucleus has slight excess surface moisture. This imparts plasticity on the nucleus and enhances particle deformations and subsequent coalescence. Another reason for the growth of agglomerates size is attributed to growth mechanisms that describe the successive addition of material on already formed nuclei.

\section{Constant Size Zone:}

In this zone agglomerates cease to grow or even show slight decrease in size. Here the frequency of coalescence is balanced by the breakage frequency of agglomeration. The size reduction may be due to attrition, breakage and shatter. The rate determining step in agglomeration growth occurs in zero growth zones when bridging liquid is squeezed out of the pores as the initial floccules are transformed into small agglomerates. The rate determining step is the collision of particle with the bridging liquid droplets prior to the formation of liquid bridges. The rate is governed by the rate of agitation. The strength of the agglomerates is determined by interfacial tension between the bridging liquid and the continuous liquid phase, contact angle and the ratio of the volumes of the bridging liquid and solid particles.

\section{EVALUATION PARAMETERS FOR CRYSTALLO- CO-AGGLOMERATES:}

\section{Micromeritic properties: ${ }^{34}$}

\section{Angle of repose:}

Flow property of the material depends on the particle size, shape, distribution, surface texture or roughness, surface area and applied force. The angle of repose can be determined by using the following equation.

$$
\operatorname{Tan} \Theta=h / 0.5 \mathrm{~d}
$$

Where h-height of the pile and d- diameter of the pile

Values for angle of repose $\leq 30$ usually indicate free flowing material and angle $\geq 40$ suggested a poor flowing material.

\section{Compressibility or Carr's index:}

The ease with which a material can be induced to flow is given by application of compressibility index.

$$
\mathrm{I}=\left(1-\mathrm{v} / \mathrm{v}_{\mathrm{o}}\right) * 100
$$

Where $\mathrm{v}=$ the volume occupied by a sample of the powder after being subjected to a standardized tapping procedure and $\mathrm{v}_{\mathrm{o}}=$ the volume before tapping.

\section{Hausner ratio:}

It is calculated from bulk and tapped densities.

Hausner ratio = tapped density / bulk density

Values less than 1.25 indicates good flow (20\% Carr's index) and the value greater than 1.25 indicates poor flow (33\% Carr's index). If it is between 1.25-1.5 add the glident to improve flow.

\section{Mechanical properties: ${ }^{35}$}

Tensile strength of spherical agglomerates is determined by compressing $500 \mathrm{mg}$ of crystals using hydraulic press at different forces $(\mathrm{kg} / \mathrm{cm} 2)$ for $1 \mathrm{~min}$. The hardness of each compact is measured using Pfizer hardness tester.

\section{Dissolution studies:}

Dissolution of spherical agglomerates is determined by using the official dissolution apparatus and comparative studies are done for agglomerated crystals and non agglomerate. Dissolution rate and bioavailability depends on the particle size and density and specific surface area of the agglomerated crystals.

\section{Solubility studies:}

Solubility studies are carried out in distilled water and dissolution medium by using Flask shaker method.

Spherical agglomerated crystals are introduced into a flask 
containing distilled water and dissolution medium. The flasks are shaken for 24 hours at room temperature. The filtrates are then diluted with the respective medium and content is determined by a suitable analytical method.

\section{Particle Size and Size Distribution:}

Size of the particle and their distributions can be determined by simple sieve analysis with the help of a RoTap sieve shaker.

\section{Optical microscopy:}

The shape of spherical agglomerates is studied by observing them under optical microscope.

\section{Differential scanning calorimeter (DSC):}

DSC measures the heat loss or gain resulting from physical or chemical changes within a sample. It is also useful to determine thermal degradation, purity, polymorphism and drug-excipient compatibility.

\section{X-ray powder diffraction:}

Each diffraction pattern is characteristics of a specific crystalline lattice for a given compound. The form of crystals in agglomerates is determined by using X-ray

\section{REFERENCES:}

1. Patil SV, Sahoo SK. Spherical Crystallization: a Method to Improve Tabletability. Research J. Pharm. and Tech.2 (2): April.June. 2009.

2. Ali Nokhodchi, Maryam Maghsoodi, Davood Hassan-Zadeh, Mohammad Barzegar Jalali. Preparation of agglomerated crystals for improving flowability and compactibility of poorly flowable and compactible drugs and excipients. Powder Technology (2007) $17573-81$.

3. A. Hassanpour, M. Ghadiri, DEM simulation and experimental evaluation of heckle analysis of bulk powder compression, Powder Technology. 141 (2004) 251-261.

4. J. Denny, Compaction equations: a comparison of the Heckel and Kawakita equations, Powder Technology . 127 (2002) 162-172.

5. Yadav VB , Yadav AV. Comparative Tabletting behavior of Carbamazepine granules with spherical agglomerated crystals prepared by spherical crystallization technique. International Journal of ChemTech Research CODEN( USA): IJCRGG ISSN : 0974-4290 July-Sept 2009 Vol.1 No.3 476-482.

6. Kadam SS, Mahadik KR, Paradkar AR, inventors. A process for making agglomerates for use as or in a drug delivery system. Indian patent 183036 . February 14, 1997.

7. Kadam SS, Mahadik KR, Paradkar AR, inventors. A process for making agglomerates for use as or in a drug delivery system. Indian patent 183481. February 14, 1997.

8. Atmaram P. Pawar, Anant R. Paradkar,Shivajirao S. Kadam, and Kakasaheb R. Mahadik. Crystallo-co-agglomeration: A Novel Technique To Obtain Ibuprofen-Paracetamol Agglomerates. AAPS PharmSciTech 2004; Article 445 (3).

9. Sarfaraz Md., Arshad Ahmed Khan K., Doddayya H., Reddy S.R. and Udupi R.H. Particle Design of Aceclofenac-Disintegrant Agglomerates for Direct Compression by Crystallo-CoAgglomeration Technique Asian J. Pharm. Tech. 2011; Vol. 1: Issue 2, 40-48.

10. A. Nokhodchi and M. Maghsoodi. Preparation of Spherical Crystal Agglomerates of Naproxen Containing Disintegrant for Direct Tablet Making by Spherical Crystallization Technique. AAPS PharmSciTech, March 2008 Vol. 9, No. 1.

11. Patil S. V., Sahoo S. K. Pharmaceutical overview of spherical crystallization Research Library, Der Pharmacia Lettre, 2 (1), 421-426.

12. Kawashima Y, Cui F, Takeuchi H, Hino T, Niwa T, Kiuchi K. Parameters determining the agglomeration behavior and the micrometric properties of spherically agglomerated crystals powder diffraction technique. This is an important technique for establishing batch-to-batch reproducibility of a crystalline form.

\section{CONCLUSION:}

The spherical crystallization could shorten the manufacturing process in tabletting, so that the cost and time of manufacturing of the tablets could be reduced. The spherically agglomerated crystals were successfully prepared for direct tableting by the spherical agglomeration technique. The micromeritic properties of agglomerates such as flowability, packability and compactibility were dramatically improved, resulting in successful direct tableting. The main factor in the improvement of flowability and packability was due to their spherical shapes and smooth surfaces. Therefore, from the above it can be concluded that spherical crystallization is a tool of particle engineering, which can transform the poorly flowable drug powder into spherical crystals, those are best suited for direct compression. The conversion of poorly flowable powder into spherical agglomerates enhances the speed of tableting because of elimination of most of steps, which required in the wet granulation and in dry granulation process.

prepared by spherical crystallization technique with miscible solvent system. Int J Pharm. 1995; 119, 139-147.

13. Sano A, Kuriki T, Kawashima Y, Takeuchi H, Hino T, Niwa T.Particle design of Tolbutamide by the spherical crystallization technique IV, Improved of dissolution and bioavailability of direct compression tablets prepared using Tolbutamide agglomerated crystals, Chem. Pharm. Bull. 40, 3030-3035.

14. Chavda V, Maheshwari RK. Tailoring of ketoprofen particle morphology via novel crystallo-coagglomeration technique to obtain a directly compressible material . Asian journal of Pharmaceutics. 2(1), 61-67.

15. Yadav A.V, Yadav V.B. Designing of pharmaceuticals to improve physicochemical properties by spherical crystallization technique Journal of Pharmacy Research 1(2), 105-112.

16. Anant $\mathrm{R}$ paradkar, Atmaram Pawar, Namdeo $\mathrm{R}$ Jadhav. Crystallo-co-agglomeration: A novel particle engineering technique. Asian journal of pharmaceutics.2010,vol-4 (1) 4-10.

17. Pawar AP, Paradkar AR, Kadam SS, Mahadik KR. Agglomeration of ibuprofen with talc by novel crystallo-coagglomeration technique. AAPS PharmSciTech 2004 ,5,1-6.

18. Maghsoodi M, Omid T, Martin GP, Nokhodchi A. Particle design of naproxen-disintegrant agglomerates for direct compression by a crystallo-co-agglomeration technique. Int J Pharm 2008;351:4554.

19. Jadhav NR. Studies on agglomerates of bromhexine hydrochloride loaded talc prepared by crystallo-co-agglomeration [masters thesis], University of Pune 1998.

20. Pawar AP. Studies on spherical crystallisation of various pharmaceuticals [Doctoral thesis], University of Pune; 1999

21. Limzerwala RB. Optimization of the wet agglomeration of talc [Masters thesis], University of Pune; 1996.

22. Janika Garg, Sadhna Khatry, Sandeep Arora. spherical crystallization: an overview. Sadhna Khatry et al. /International Journal Of Pharmacy\&Technology. ISSN: 0975-766X.

23. P.K. Kulkarni, B.G. Nagavi. Ind. Jr. Pharm. Eudc. 2002; 36(2), 66-73.

24. Kawashima Y, Handa T, Hirofumi T, Okumura M. Effects of polyethylene glycol on size of agglomerated crystals of phenytoin prepared by the spherical crystallization technique. Chem Pharm Bull (Tokyo) 1986;34:3403-7.

25. Kawashima Y, Handa T, Tekeuchi H, Okumara M, Katou H, Nagata O. Crystal modification of phenytoin with polyethylene glycol for improving mechanical strength, dissolution rate and 
bioavailability by spherical crystallization technique. Chem Pharm Bull (Tokyo) 1986:34,3376-83.

26. Jadhav NR, Pawar AP, Paradkar AR. Design and evaluation of deformable talc agglomerates prepared by crystallo-coagglomeration technique for generating heterogeneous matrix. AAPS PharmSciTech 2007:8,1-7.

27. Pawar AP, Paradkar AR, Kadam SS, Mahadik KR. Effect of polymers on crystallo-co-agglomeration of ibuprofen-paracetamol: Factorial design. Indian J Pharm Sci 2007:69,654-8.

28. Dubernet C, Rouland JC, Benoit JP. Ibuprofen-loaded ethylcellulose microspheres: analysis of the matrix structure by thermal analysis. J Pharm Sci. 1991;80,1029-1133.

29. Paradkar AR, Pawar AP, Chordiya JK, Patil VB, Ketkar AR. Spherical crystallization of celecoxib. Drug Dev Ind Pharm. 2002;28,1213-1220.
30. Kawashima Y, Aoki S, Takenama H, Miyake Y. Preparation of spherically agglomerated crystals of aminophylline. J Pharm Sci. 1984;73,1407-1409.

31. Ueda M, Nakamura Y, Makita H, Kawashima Y. Preparation of microcapsules masking the bitter taste of enoxacin by using one continuous process of agglomeration and microencapsulation. J Microencapsul. 1993;10,461-473.

32. Chouracia MK, Jain A, Valdya S, Jain, SK, Utilization of spherical crystallization for preparation of directly compressible materials, Indian Drugs, 2004:41(6), 319-29.

33. Patil SV, Spherical Crystallization: A Method to Improve Tabletability, Research J. Pharm. And Tech.2009;2(2), 234-237.

34. Deshpande, M. C., Mahadik, K. R., Pawar, A.P. and Paradkar, A. R., (1997). Evaluation of spherical crystallization as particle size enlargement technique for aspirin Ind.Jr.Pharm.Sci. 59(1), 32-34.

35. Jarosz, P.J. and Parrott E.L. Compression of granule strength and tablet tensile strength. Jr. Pharm. Sci. 1983, 72(5), 530-534. 